\title{
Stevens-Johnson syndrome and toxic epidermal necrolysis in an academic hospital setting: a 5-year retrospective study
}

\section{Ewa Stocka-Łabno', Natalia Gabzdyl', Magdalena Misiak-Galazka', Małgorzata Pawłowska-Kisiel', Tomasz Łazowski², Lidia Rudnicka'}

${ }^{1}$ Department of Dermatology, Medical University of Warsaw, Warsaw, Poland, ${ }^{2}$ Department of Anaesthesiology and Intensive Care, Medical University of Warsaw, Warsaw, Poland

Corresponding author: Lidia Rudnicka, MD, PhD., E-mail: lidiarudnicka@gmail.com

\begin{abstract}
Introduction: Toxic epidermal necrolysis and Stevens-Johnson syndrome are acute life-threatening mucocutaneous reactions to drugs. The aims of the study were to identify these drugs and characterize population prone to these reactions. Materials and Methods: Data including demographics, culprit drug, clinical characteristics, course of disease, treatment given, and therapeutic responses were retrospectively collected from medical records of 31 patients admitted to Department of Dermatology from January 2009 to December 2014. Results: Drugs most commonly involved in Stevens-Johnson syndrome were antimicrobials: ciprofloxacin, doxycycline, cefuroxime, trimethoprim, amoxicillin, clindamycin, co-trimoxazole (50\% of patients) and nonsteroidal anti-inflammatory drugs: ibuprofen, naproxen, metamizole, piroxicam (29\% of patients). Drugs involved in toxic epidermal necrolysis were antimicrobials: sulfasalazine, co-trimoxazole, cefuroxime, clindamycin (71\% of patients) and anticonvulsants: lamotrigine (29\% of patients). The comorbidities' characteristic for the group of patients affected by toxic epidermal necrolysis were psychiatric and autoimmune disorders. The most common complication was infection. Two patients died and in both cases the cause of death was sepsis. Conclusions: The study indicates that in observed population drugs with the highest risk of most severe reactions are lamotrigine (anticonvulsant) and antimicrobials (most commonly sulfonamides), therefore it is advisable to consider carefully administration of these drugs, especially to patients with history of autoimmune reactions.
\end{abstract}

Key words: Stevens-Johnson syndrome; Toxic epidermal necrolysis; Adverse drug reaction; Retrospective study

\section{INTRODUCTION}

Toxic epidermal necrolysis (TEN) and Stevens-Johnson syndrome (SJS) are acute life-threatening mucocutaneous reactions usually to drugs characterized by extensive necrosis and detachment of the epidermis plus widespread erythematous or purpuric macules or flat atypical lesions [1] (Fig. 1).

These two conditions are severity variants of an identical process of epidermal necrolysis (EN) that differs only in the final extent of body surface involved: below $10 \%$ in SJS, from 10 to $30 \%$ in overlap SJS/TEN and above $30 \%$ of the body surface in TEN $[1,2]$.

$\mathrm{EN}$ is a rare condition. The overall incidence of SJS and TEN was estimated at 1 to 6 cases per million person-years and 0.4 to 1.2 cases per million personyears, respectively $[1]$.

The skin reactions are mediated by cytotoxic T-cells activated in response to the culprit drug. Among more than 100 different drugs implicated, the associated with the highest risk are: antibacterial sulfonamides, aromatic anticonvulsants, allopurinol, oxicam nonsteroidal

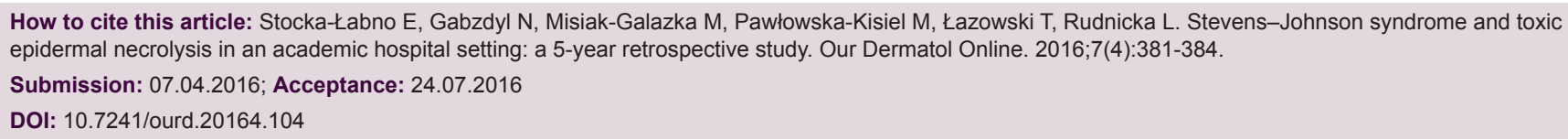




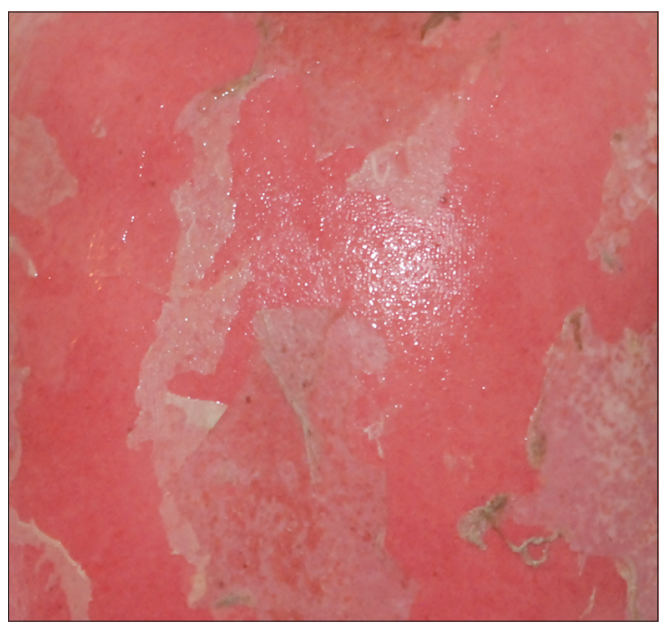

Figure 1: Detachment of the epidermis in toxic epidermal necrolysis. By courtesy of Marek Opala.

anti-inflammatory drugs (NSAIDs), lamotrigine, and nevirapine $[1,3]$.

The treatment is based on prompt withdrawal of the culprit drug and supportive care similar to burns treatment. It is advisable to transfer patients with extensive and rapidly progressing reactions to an intensive care unit (ICU) or a burn centre. Steroids administration is controversial due to their expected effect of decreasing the adverse reaction on one hand and increasing susceptibility to infection on the other hand. Other medicaments considered are: cyclosporine A, intravenous immunoglobulin (IVIG), anti-TNF. Pharmacotherapy includes antibiotics when infection is suspected $[1,3]$.

The main aims of the study were to identify drugs with the highest risk of causing most severe adverse reactions and to attempt to characterize groups of patients with high risk of developing EN.

\section{MATERIALS AND METHODS}

The medical records of SJS/TEN patients hospitalized at Department of Dermatology in The Infant Jesus Teaching Hospital in Warsaw, Poland between 2009 and 2014 were retrospectively reviewed. Based on the Algorithm for Assessment of Drug Causality in Stevens-Johnson Syndrome and Toxic Epidermal Necrolysis (Alden) [4] in 31 patients drug causality was established. Other causes of skin reactions observed among those patients were excluded as less probable.

Among patients with drug induced SJS/TEN the following variables were observed: patient characteristics (age, gender, ethnicity, comorbidities), culprit drug, time interval between drug intake and onset of symptoms (latent period), drug reaction observed, factors associated with severity of illness (SCORTEN) [5], systemic complications, length of hospital stay, treatment given, and outcomes. The SCORTEN scale includes: age, malignancy, tachycardia, initial percentage of epidermal detachment, serum urea, serum glucose and bicarbonate. Since bicarbonate levels are not routinely determined in our department, that value was excluded and modified score for toxic epidermal necrosis (mSCORTEN) was calculated $[5,6]$.

\section{RESULTS}

Twenty four patients were diagnosed with drug associated SJS and 7 with TEN over a 5-year study period.

\section{Patient Characteristics}

The patients' age ranged from 19 to 87 years, with a median of 41 years. The gender distribution was 20 females (65\%) to 11 males (35\%), but the difference was not statistically significant (two-sided p-value $=$ $0.074)$.

Twenty nine patients were Polish, one Russian, one Ukrainian. All patients were Caucasian.

A total number of eighteen (75\%) patients with SJS had comorbidities. The most common comorbidities were hypertension (20\%), asthma and allergies (ingested, contact and inhaled allergy) (16\%), thyroid diseases (16\%). All of TEN patients (7/7) had comorbidities including: psychiatric disorders (bipolar affective disorder, paranoid schizophrenia (43\%)), autoimmune disorders (ankylosing spondylitis, psoriasis vulgaris, rheumatoid arthritis, Hashimoto's thyroiditis (57\%)), asthma and allergies (43\%), or hypertension (43\%).

\section{Culprit Drugs (Table 1)}

SJS was caused most frequently by antimicrobials (50\% of patients) and NSAIDs (29\% of patients). In other cases the culprit drugs were: antifungal medication (two patients), angiotensin-converting enzyme (ACE) inhibitor (one patient), carbamazepine (one patient) and unithiol (one patient).

Most common causative agents in TEN were likewise antimicrobials (71\%), especially sulfonamides and 
anticonvulsant - lamotrigine (29\%).

The latent period ranged from 1 to 30 days. In $40 \%$ of cases it was shorter than 5 days, in $55 \%$ between 5 and 28 days and in $4 \%$ longer than 28 days. The average latent period was 7 days. NSAIDs had the average latent period of 3 days, antimicrobials of 8 days, antifungals 1 day and anticonvulsants 12 days.

\section{Complications, Length of Stay and Outcomes}

Fifteen patients (48\%) had increased levels of inflammatory markers including $\mathrm{C}$-reactive protein (CRP), erythrocyte sedimentation rate (ESR) or white blood cell count (WBCs). Six of them had

Table 1: Culprit drugs

\begin{tabular}{lcc}
\hline Drug (s) & $\begin{array}{c}\text { Nr of SJS } \\
\text { cases }\end{array}$ & $\begin{array}{c}\text { Nr of TEN } \\
\text { cases }\end{array}$ \\
\hline Antimicrobials & 12 & 5 \\
Ciprofloxacin & 2 & \\
Doxycycline & 2 & 1 \\
Clindamycin & 1 & \\
Amoxicillin & 1 & \\
Amoxicillin and Clavulanate & 1 & 1 \\
Cefuroxime & 2 & \\
Trimethoprim & 2 & 1 \\
Sulfamethoxazole+trimethoprim & 1 & \\
Sulfasalazine & & \\
NSAIDs & 7 & \\
Naproxen & 1 & \\
Ibuprofen & 4 & \\
Metamizole & 1 & \\
Piroxicam & 1 & \\
Antifungals & 2 & \\
Fluconazole & 1 & \\
Miconazole & 1 & \\
Anticonvulsants & 1 & \\
Carbamazapine & 1 & \\
Lamotrigine & & \\
Others & 1 & \\
Enalapril & & \\
Unithiol & 1 & \\
\hline
\end{tabular}

neutrophilia. Three patients (10\%) had transaminitis. One case of SJS was complicated by bacterial conjunctivitis. Additionally two cases of TEN had vascular complications: thrombophlebitis and varicose vein bleeding.

The average length of stay of SJS and TEN patients on the dermatology unit was 7.5 days and 16.3 days, respectively.

\section{There were no Cases of Mortality among SJS Patients}

Three TEN patients (43\%) were transferred to ICU (Intensive Care Unit). Two of them died of sepsis caused by Methicillin-resistant Staphylococcus aureus (MRSA) and Extended spectrum beta-lactamase (ESBL)-producing bacteria. One of the patients who died had high (3) and the other low (1) MSCORTEN factor at the time they had been admitted to hospital (Table 2).

\section{Treatment}

Twenty SJS patients received systemic steroids, 18 topical steroids, 18 antihistamines and 11 antibiotics whereas 5 TEN patients received systemic steroids, 5 cyclosporine, 6 antibiotics, 2 IVIG and 1 plasmapheresis. The most intensive treatment (IVIG, cyclosporine, systemic steroids and antibiotics) received the two patients with reaction to anticonvulsants (lamotrigine).

\section{DISCUSSION}

The analysis of the population of patients shows that the SJS has much higher morbidity rate than TEN (24 SJS to 7 TEN patients). In both groups patients fall into two age categories: from 19 to 44 and from 57

Table 2: Values of mSCORTEN among TEN patients. One point is given for each of six criteria present at the time of admission (age $>40$ years, associated malignancy, heart rate $>120$ beats $/ \mathrm{min}$, detached body surface $>10 \%$, serum BUN $>27 \mathrm{mg} / \mathrm{dl}$, serum glucose $>250 \mathrm{mg} / \mathrm{dl}$ )

\begin{tabular}{|c|c|c|c|c|c|c|c|c|c|}
\hline Drug & Age & Cancer & $\begin{array}{l}\text { Heart rate } \\
\text { (BPM) }\end{array}$ & $\begin{array}{c}\text { Detached } \\
\text { body } \\
\text { surface } \\
(\%)\end{array}$ & $\begin{array}{l}\text { Serum } \\
\text { BUN } \\
\text { (mg/dL) }\end{array}$ & $\begin{array}{l}\text { Serum } \\
\text { glucose } \\
\text { (mg/dL) }\end{array}$ & $\begin{array}{l}\text { mSCORTEN } \\
\text { value }\end{array}$ & $\begin{array}{c}\text { Risk of } \\
\text { mortality } \\
(\%)\end{array}$ & Outcomes \\
\hline Lamotrigine & 34 & No & 85 & $>10$ & 20 & 118 & 1 & 3-12 & \\
\hline Lamotrigine & 34 & No & 88 & $>10$ & 19 & 135 & 1 & $3-12$ & Transfer to ICU, deatr \\
\hline Sulfasalazine & 37 & No & 120 & $>10$ & 28 & 113 & 3 & $35-58$ & Transfer to ICU \\
\hline Cefuroxime & 71 & No & 78 & $>10$ & 88 & 159 & 3 & $35-58$ & Transfer to ICU, deatr \\
\hline Clindamycin & 76 & No & 75 & $>10$ & 53 & 109 & 3 & $35-58$ & \\
\hline Sulfasalazine & 30 & No & 70 & $>10$ & 11 & 84 & 1 & $3-12$ & \\
\hline Sulfamethoxazole+trimethoprim & 85 & No & 76 & $>10$ & 38 & 119 & 3 & $35-58$ & \\
\hline
\end{tabular}


to 87 years. The most common comorbidities in SJS group (hypertension, allergy, thyroid disease) closely mirror those in general population though the share of comorbidities with immune system malfunction draws attention.

The comorbidities characteristic for the TEN group are psychiatric and autoimmune disorders.

Due to genetic factors the epidemiology and causative drugs differ significantly in different ethnic populations $[5,6]$.

In our study drugs most commonly involved in SJS were antimicrobials and NSAIDSs and in TEN antimicrobials and anticonvulsants which corresponds with results of other European studies [7,8]. Most of these drugs are evaluated as high or significant risk drugs in EuroSCAR study [9]. The culprit drugs in case of the two patients who died were lamotrigine and cefuroxime.

The most common complication was infection. Among TEN patients only one out of seven did not require antibiotics. In two cases the infection led to death.

So far no single major HLA-related genetic risk factor was identified for lamotrigine-induced severe cutaneous adverse reactions (SCARs) among patients of European origin [10]. However success in establishing correlation between reactions to carbamazepine and HLA-B*1502 allele [11] encourages continuation of the studies.

\section{CONCLUSION}

The study indicates that in observed population the drugs with the highest risk of most severe reactions are lamotrigine and antimicrobials (most commonly sulfonamides). Due to cases of mortality and necessity of long hospitalization and expensive treatment it is advisable to consider carefully administration of these drugs especially to patients with history of autoimmune reactions.

\section{REFERENCES}

1. Valeyrie-Allanore LL, Roujeau J. Chapter 40. Epidermal Necrolysis (Stevens-Johnson Syndrome and Toxic Epidermal Necrolysis). In: Fitzpatrick's Dermatology in General Medicine, 8e. Goldsmith LA, Katz SI, Gilchrest BA, Paller AS, Leffell DJ, Wolff K. (eds). NY: McGraw-Hill, New York. 2012: 439-48.

2. Bastuji-Garin S, Rzany B, Stern RS, Shear NH, Naldi L, Roujeau JC. Clinical classification of cases of toxic epidermal necrolysis, Stevens-Johnson syndrome, and erythema multiforme. Arch Dermatol. 1993;129:92-6.

3. Mockenhaupt M. Severe cutaneous adverse reactions. In: Braun-Falco's Dermatology. Burgdorf W, Plewig G, Wolff H, Landthaler M, (eds). Springer, Heidelberg. 2009: 473-84.

4. Sassolas B, Haddad C, Mockenhaupt M, Dunant A, Liss Y, Bork K, et al. ALDEN, an Algorithm for Assessment of Drug Causality in Stevens-Johnson Syndrome and Toxic Epidermal Necrolysis: Comparison With Case-Control Analysis. Clin Pharmacol Ther. 2010;88:60-8.

5. Bastuji-Garin S, Fouchard N, Bertocchi M, Roujeau JC, Revuz J, Wolkenstein P. SCORTEN: a severity-of-illness score for toxic epidermal necrolysis. J Invest Dermatol. 2000;115:149-53.

6. Sun J, Liu J, Gong Q-L, Ding GZ, Ma LW, Zhang LC, et al. StevensJohnson Syndrome and toxic epidermal necrolysis: a multi-aspect comparative 7-year study from the People's Republic of China. Drug Des Devel Ther. 2014;8:2539-47.

7. Weinand C, Xu W, Perbix W, Lefering R, Maegele M, Rathert M, et al. 27 years of a single burn centre experience with Stevens-Johnson syndrome and toxic epidermal necrolysis: analysis of mortality risk for causative agents. Burns. 2013;39:1449-55.

8. Dao R-L, Su S-C, Chung W-H. Recent advances of pharmacogenomics in severe cutaneous adverse reactions: immune and nonimmune mechanisms. Asia Pacific Allergy. 2015;5:59-67.

9. Mockenhaupt M, Viboud C, Dunant A, Naldi L, Halevy S, Bouwes Bavinck JN, et al. Stevens-Johnson syndrome and toxic epidermal necrolysis: assessment of medication risks with emphasis on recently marketed drugs: the EuroSCAR-study. J Invest Dermatol. 2008;128:35-44.

10. Kazeem GR, Cox C, Aponte J, Messenheimer J, Brazell C, Nelsen AC, et al. High-resolution HLA genotyping and severe cutaneous adverse reactions in lamotrigine-treated patients. Pharmacogenetics and Genomics. 2009;19:661-5.

11. Tangamornsuksan W, Chaiyakunapruk N, Somkrua R, Lohitnavy M, Tassaneeyakul W. Relationship between the HLA-B*1502 allele and carbamazepine-induced Stevens-Johnson syndrome and toxic epidermal necrolysis: a systematic review and meta-analysis. JAMA Dermatol. 2013;149:1025-32.

Copyright by Ewa Stocka-Łabno, et al. This is an open access article distributed under the terms of the Creative Commons Attribution License, which permits unrestricted use, distribution, and reproduction in any medium, provided the original author and source are credited.

Source of Support: Nil, Conflict of Interest: None declared. 